\title{
Análisis de la capacidad de medición de una máquina herramienta usando el método Monte Carlo para estimar la incertidumbre
}

\author{
Pablo Pérez ${ }^{1}$, Sergio Aguado ${ }^{2}$, José Antonio Albajez ${ }^{1}$, Jorge Santolaria ${ }^{1}$ \\ ${ }^{1}$ Grupo de Ingeniería de Fabricación y Metrología Avanzada (GIFMA) \\ Instituto de Investigación en Ingeniería de Aragón (I3A) \\ Universidad de Zaragoza, Mariano Esquillor s/n, 50018, Zaragoza, Spain. \\ Tel. +34-976762707, e-mail: pperezm@unizar.es \\ ${ }^{2}$ Centro Universitario de la Defensa (Universidad de Zaragoza).
}

\begin{abstract}
Con Máquina Herramienta $(\mathrm{MH})$ se realiza la fabricación de piezas que requieren alta precisión, sin embargo, la verificación suele realizarse en Máquinas de Medición por Coordenadas (MMC). Actualmente las $\mathrm{MH}$ pueden realizar tareas de medición mediante palpador. Para que estas mediciones sean trazables, es necesario conocer la incertidumbre de medida.
\end{abstract}

\section{Introducción}

Realizar la verificación de un pieza en proceso, mientras la pieza sigue amarrada a la $\mathrm{MH}$, permite conservar el sistema de coordenadas para posibles re-trabajos que haya que realizar, consiguiendo de así un ahorro del tiempo de fabricación, de material y reducción de costes. Para alcanzar este objetivo es necesario incorporar técnicas de metrología dimensional trazable en la $\mathrm{MH}$ para que el programa de fabricación resultante produzca la pieza deseada dentro de las tolerancias especificadas [1].

Los errores geométricos de una $\mathrm{MH}$ son la diferencia entre el trayecto que realiza la herramienta y el trayecto deseado, estos se pueden medir individualmente para cada eje y posición del espacio de trabajo (método directo) o se puede obtener la influencia conjunta de todos los errores (método indirecto). La norma UNE-ISO 230-1:2014 [2] especifica la metodología para medición directa de errores. Mientras que la verificación volumétrica mediante laser tracker es el método más utilizado para medición indirecta [3].

La dispersión de los errores geométricos junto con otras fuentes de error que puedan afectar a la medición, caracterizan el valor de incertidumbre. La incertidumbre de medición representa el intervalo en el que es más probable encontrar el valor real de una medida. La Guía para la Expresión de la Incertidumbre de Medida (GUM) [4] proporciona el marco básico para evaluar la incertidumbre, pero no funciona correctamente en un procesos no lineales como el obtenido en una calibración basada en verificación volumétrica, por lo que se recomienda usar el método Monte Carlo.

\section{Metodología}

La metodología ha sido definida para una $\mathrm{MH}$ de 3 ejes con configuración XYFZ (Figura 1), aunque es extrapolable para otros tipos de $\mathrm{MH}$. El objeto medido es una placa de agujeros calibrada hecha de aluminio. Dicha placa es medida simultáneamente por la $\mathrm{MH}$ mediante un palpador y con un Laser Tracker cuyo retro-reflector se ha adherido magnéticamente al palpador.

La estrategia de medición consiste en medir 28 agujeros con una trayectoria en espiral para reducir la influencia del juego de la MH (Figura 2). En cada agujero se miden 4 puntos para calcular el centro del círculo por mínimos cuadrados. A las coordenadas de los centros obtenidas se les compensa el desalineamiento y la expansión térmica, ya que estos errores no son de la MH, sino del patrón.

Con las coordenadas medidas, las coordenadas nominales y el modelo cinemático de la $\mathrm{MH}$ se calculan las funciones de aproximación de los errores geométricos.

Se ha desarrollado un software que introduce en el modelo cinemático el ruido del Laser Tracker y calcula las funciones de aproximación. Se han simulado 1000 ensayos donde el ruido del Laser Tracker ha sido modelado como una distribución normal con $24 \mu \mathrm{rad}$ para los encoders angulares y 4 $\mu \mathrm{m} \pm 0.8 \mu \mathrm{m} / \mathrm{m}$ para el error radial. La Figura 3 muestra el valor del error volumétrico residual para los tres ejes y el error en distancia total resultante después de realizar la optimización no lineal. 
El valor medio del error en distancia es considerado como un error sistemático que hay que compensar a la hora de ofrecer el resultado de la medición y cuyo valor es 12,34 $\mu \mathrm{m}$. Mientras que la desviación estándar, que es de 7,06 $\mu \mathrm{m}$, representa la componente de incertidumbre asociada al proceso de medición $\left(u_{p}\right)$. Esta componente junto a la incertidumbre de calibración $\left(u_{c a l}\right)$, la incertidumbre asociada al error sistemático $\left(u_{b}\right)$ y la incertidumbre asociada a variaciones del material $\left(u_{w}\right)$, componen la incertidumbre expandida.

$$
U=k \cdot \sqrt{u_{c a l}^{2}+u_{p}^{2}+u_{b}^{2}+u_{w}^{2}}
$$

La Figura 4 muestra el valor de la incertidumbre expandida $(U)$ en el espacio de trabajo, con un nivel de confianza del 95\% $(k=2)$.

\section{Conclusiones}

Cuando la MH se utiliza como sistema de medición es necesario determinar la incertidumbre de medida para que la medición tenga trazabilidad. Se han realizado tests que demuestran la influencia del ruido del Laser Tracker en una verificación

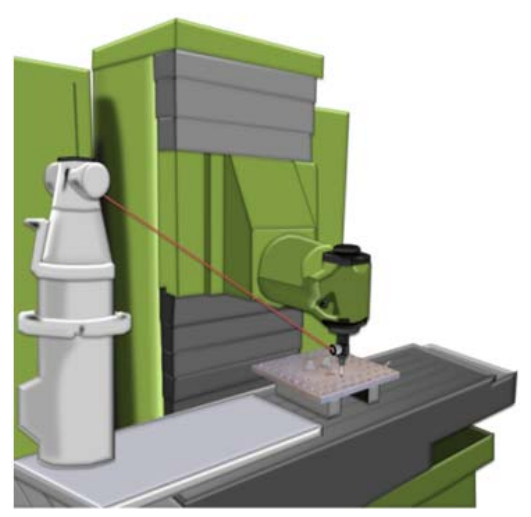

Fig. 1. Configuración del ensayo.

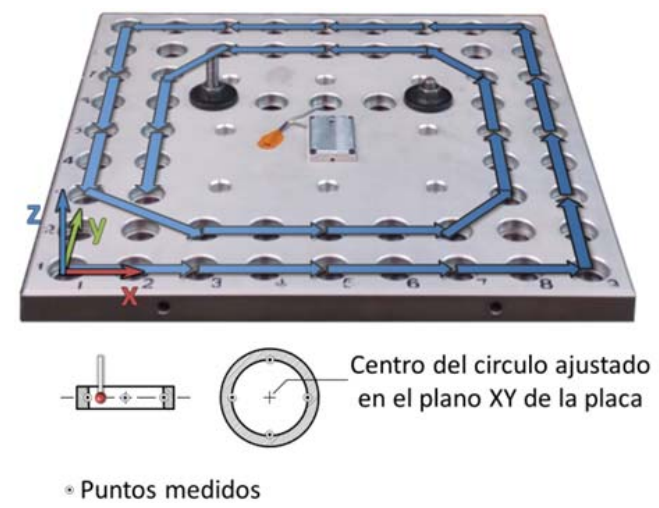

Fig. 2. Estrategia de medición del objeto calibrado. volumétrica. A los valores de una medición real se les ha añadido una simulación del ruido para obtener 1000 mediciones y por lo tanto 1000 funciones de error. Con estas simulaciones se ha obtenido el error sistemático que comete la $\mathrm{MH}$ y la incertidumbre del proceso de medición.

\section{REFERENCIAS}

[1]. LONGSTAFF, A.P., FLETCHER, S., and PARKINSON, S. The role of measurement and modelling of machine tools in improving product quality. Int. J. Metrol. Qual. Eng. 2013, 4, 177-184. Available from: doi: 10.1051/ijmqe/2013054.

[2]. UNE-ISO 230-1:2014 Test code for machine tools Part 1: Geometric accuracy of machines operating under no-load or quasi-static conditions.

[3]. AGUADO, S., SANTOLARIA, J., SAMPER, D., AGUILAR, J.J., and VELÁZQUEZ, J. Improving a real milling machine accuracy through an indirect measurement of its geometric errors. Int. Journal of Manufacturing Systems. 2016, 40, 26-36. Available from: doi: 10.1016/j.jmsy.2016.05.006

[4]. Evaluation of measurement data: Guide to the expression of Uncertainty in Measurement (GUM). JCGM 100:2008.
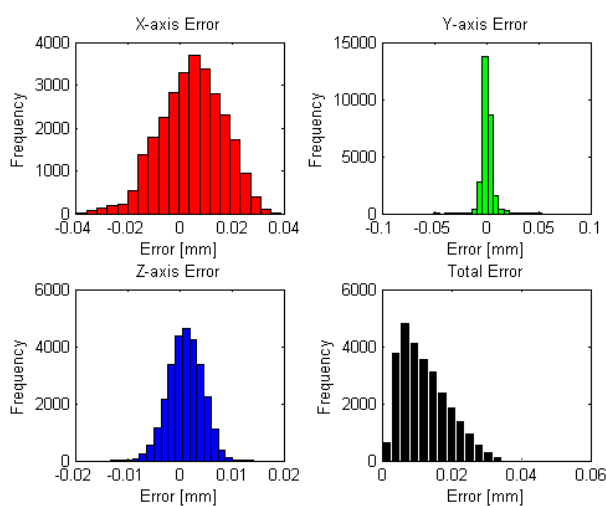

Fig. 3. Distribución del error residual después de optimizar.

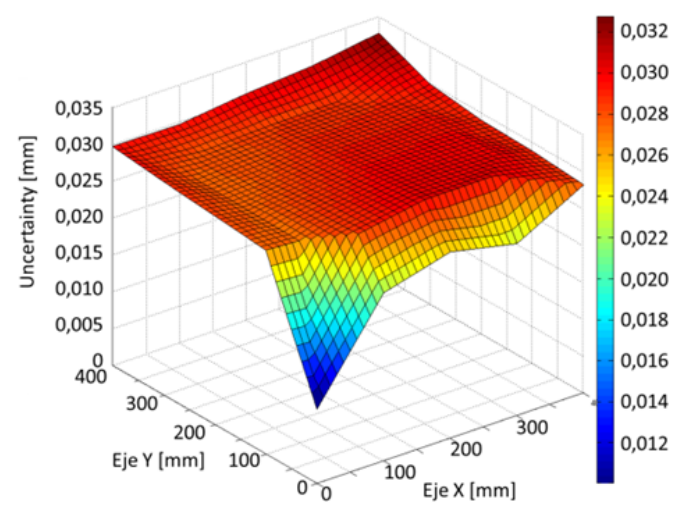

Fig. 4. Incertidumbre expandida del área de trabajo. 